\author{
Military Technical College \\ Kobry El-Kobbah, \\ Cairo, Egypt.
}

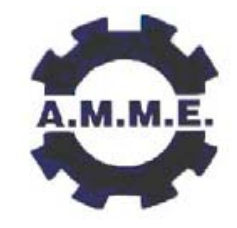

\title{
VIBRATION SOURCE ESTIMATION INSIDE OF MACHINERY
}

\author{
FOULADI* M.H., NOR ${ }^{* *}$ M.J.M., ARIFFIN** A.K. and ABDULLAD*** ${ }^{* *}$.
}

\begin{abstract}
In this paper, signal processing is integrated with inverse techniques to solve inverse problem for internal parts of machinery in which, sources are unknown and inaccessible. Explosions of an internal combustion engine produce powerful pulses of energy which cause the engine vibration as a response. Attenuation of engine vibration is one of the important problems for NVH engineers. Engine vibration can affect the whole chassis vibration and interior noise level. Engine vibration is the dominant source of noise emissions (less than $200 \mathrm{~Hz}$ ) inside the vehicle compartment. In this research, inverse techniques are implemented to estimate unknown amount of vibration sources. Tikhonov regularization carried out to stabilize solution of ill-posed problem. L-curve method is also utilised to find the regularization parameter.

In comparison with transfer path analysis, this method doesn't need to separate the observation part from the system. Despite the coherence based methods, it is practical in low frequency analysis of engine with missing inputs and outputs.
\end{abstract}

\section{KEY WORDS}

Vibration source estimation; Inaccessible sources; Inverse technique; Picard condition; L-curve; Tikhonov regularization.

* PhD Candidate, Dep. of Mech. Eng, Faculty of Eng., Universiti Kebangsaan Malaysia.

** Professor, Dep. of Mech. Eng, Faculty of Eng., Universiti Kebangsaan Malaysia.

*** Assoc. Professor, Dep. of Mech. Eng, Faculty of Eng., Universiti Kebangsaan Malaysia. 


\section{INTRODUCTION}

\section{Inverse Problems and ill Conditioning}

In some engineering problems, the governing equation, the initial and boundary condition and all material properties related to the equation are known. This is a direct problem which the engineer computes values of the field variable. In an indirect or inverse problem, one part of the usual direct problem is missing, so an experiment is needed to solve the problem and obtain information regarding the missing part in the inverse problem [1]. For an inverse input problem, the output and parameters of the system are known and the goal is to find the input of the problem. Consider Eq. (1), an inverse input problem is to find the input spectrum $X j$ from the output spectrum $Y i$ and matrices of transfer functions $\mathrm{Hij}$ :

$$
Y_{i}=\sum_{j} H_{i j} X_{j}
$$

At the first glance, it seems to be an easy task which can be carried out through multiplying the inverse of transfer functions by the output spectrum. This is the case in single input problems but some precautions should be considered before inverting the transfer functions in multiple input problems. For a square and symmetric transfer matrix of $n \times n$ size, the condition number is defined as Eq. (2) [2]:

$$
k=\frac{\max \left(\lambda_{1}, \lambda_{2}, \ldots, \lambda_{n}\right)}{\min \left(\lambda_{1}, \lambda_{2}, \ldots, \lambda_{n}\right)}
$$

where $\lambda_{1, . .,} \lambda_{n}$ are eigenvalues of the transfer matrix and can be derived implementing the singular value decomposition (SVD) technique as below:

$H=U \Sigma V^{T}$

In Eq. (3), $U$ and $V$ are left and right matrices of eigenvectors respectively and $\Sigma$ is the diagonal matrix of eigenvalues which $\lambda_{1}, \lambda_{2}, \ldots, \lambda_{n}$ are the elements on the main diagonal. A very large condition number in a system indicates that the outputs of the system are not sensitive to at least one of the inputs. Or some linear dependencies are introduced between columns of the transfer matrix. This system is called ill-conditioned and solutions are unstable in which a slight change in the input data brings a large change to the system response [1]. Regularization method is needed to avoid unstable solutions and will be discussed next.

\section{Tikhonov Regularization}

The basis of regularization methods is to add a choice criterion based on the norm of the solution. Tikhonov regularization is the most commonly used method of regularization for ill-posed problems. Consider Eq. (4) as an ill-conditioned linear system:

$A x=b$

This system can be replaced by a problem of looking for an $x$ to minimize Eq. (5): 


$$
\|A x-b\|_{2}^{2}+\lambda^{2}\|x\|_{2}^{2}
$$

This is the standard form of Tikhonov regularization where $\|\cdot\|_{2}$ is the Euclidean norm and $\lambda>0$ is the regularization parameter. The solution to this problem $x_{\text {reg }}$ is called the regularized solution [3]. It is based on the tuning of the regularizing parameter $\lambda$ which adjusts the importance given to the minimization of the residue norm $\|A x-b\|_{2}$, in one hand, and the solution norm $\|x\|_{2}$ on the other hand. The most suitable graphical tool for such analysis is L-curve that displays the compromise between minimization of these two quantities. It is plotted in log-log scale which the vertical axis is the norm of the regularized solution and the horizontal axis is the residual norm. Then it is possible to find an estimation to the optimum regularization parameter by locating the corner of the L-curve (point with maximum curvature) [4].

\section{METHODOLOGY}

\section{Experiment Setup}

Vehicle crankshaft was considered as a system. Piston movements are sources of vibration impacting the crankpins through the connecting rods. Bearings of crankshaft are nearest accessible points to sources which can be utilised to measure the response. Transfer matrices are showing characteristics of the system. They were calculated using experimental modal analysis of the crankshaft.

Measurements of response signals were carried out over a Proton Gen2 at neutral gear. Bruel \& Kjaer portable and multi-channel PULSE analyser type 3560D with triaxial accelerometer type 4506B and ENDEVCO isotron accelerometers (uni-axial) type 751-100 were utilised in the measurement devices. The Bruel \& Kjaer Pulse LabShop was the measurement software and the B \& K calibration exciter type 4294 was implemented to calibrate the accelerometers. Accelerometers were connected to crankshaft bearings as presented in Fig. 1. The triaxial accelerometer was connected to bearing no.1 (farthest to gearbox) to measure responses in three directions. For the rest of bearings, uni-axial accelerometers were utilised to measure the vibration signals in vertical direction. All accelerometers were mounted at the same position as they were used for the transfer matrix measurements in modal analysis. Hence it was applicable to implement these matrices for the current research. FFT analyser sampled the frequency domain data with a sampling frequency of $8.2 \mathrm{kHz}$ through the frequency span of $3.2 \mathrm{kHz}$. The frequency range of interest was $0-3 \mathrm{kHz}$ equal to the minimum dynamic range of accelerometers implemented in the experiment (corresponds to the dynamic frequency range of $y$ and $z$ directions of the triaxial accelerometer).

\section{Inverse Problem Formulation}

Bearing responses together with transfer matrices were utilised to formulate the inverse problem. Thikhonov regularization conducted to stabilize the ill-conditioned problem and L-curve used to find the optimum regularization parameter. Initially, regularized solutions together with transfer functions were utilised in a forward problem formulation 
to estimate the amount of responses. Thereafter, a comparison was carried out between the measured and estimated responses. Next, pressure sensors were used to measure pressure inside the cylinders. Then crankpin forces were calculated using the measured pressure values. These forces were then compared to those acquired through the inverse formulation.

\section{RESULTS AND OBSERVATIONS}

Condition number is a measure of solution's sensitivity to perturbations in the transfer matrix. As presented in Fig. 2, condition numbers of transfer matrices are very high at some frequencies and they are ill-conditioned.

\section{Singular Vector Oscillations}

Hansen [5] mentioned that the left and right singular vectors tend to have more oscillations as the index of transfer matrix increases. The high spatial frequency information is related to left and right singular vectors having high index. The vectors with small index correspond to slowly varying functions having few sign changes. If only a few of the first singular vectors are taken into account, a very smooth approximation to solution would be calculated. Because the first singular vectors are related to slowly varying functions. Also if too many singular vectors are taken into account, errors will dominate the solution. How many of these singular vectors to take into account are basically related to the level of errors contaminating the data and should be determined by regularization parameter. In other word, components corresponding to high indices (very small singular values), compared to $\sigma_{1}$ (first singular value), will hardly contribute to the transfer matrix and should be filtered out by regularization. Sign changes of the imaginary parts of the first and last index of left singular vectors for $8 \times 4$ transfer function which indicates the index values and sign changes are shown in Fig. 3.

\section{Picard Condition}

In order to have a physical solution to the inverse problem, the Fourier coefficients of the exact right hand side $\left|u_{i}^{T} b\right|$ should decay faster than the singular values $\left(\sigma_{i}\right)[5]$. These coefficients are plotted using the function "picard" from the Regularization Tools Matlab $^{\circledR}$ package [4] and is shown in Fig. 4. It is a tool for visual inspection of the problem; determines that which components will be included in the regularized solution. For this plot, all the Fourier coefficients satisfy the Picard condition and no regularization of the problem is needed. The L-curve plot for the Picard condition of Fig. 4 is calculated using the function "I_curve" of the Regularization Tools and shown in Fig. 5. The corner of the L-curve is computed at the point where the maximum curvature exists. The vertical part of the L-curve is contributed to the solution norm. From the Picard plot, an L-curve without any vertical part is expected since the solution coefficients $\left|u_{i}^{T} b\right| / \sigma_{i}$ do not increase significantly.

Another example of Picard condition is plotted in Fig. 6 where solution coefficients have significant increase and the Fourier coefficients level off at $i=3$. It means that for $i>3$ the Fourier coefficient is dominated by noise and cannot be recovered. Then problem needs regularization and only 3 singular values are expected to include in the 
regularized solution. The steady increase in the solution norm is now seen as a vertical part in the L-curve as shown in Fig. 7. A visible corner is separating a horizontal part from the vertical part.

\section{Regularization}

The transfer matrices together with measured responses were imported into the Matlab ${ }^{\circledR}$ package [4]. For each excitation, optimum regularization parameter at each frequency was found using the L-curve criterion. In the L-curve, the flat horizontal part is characterized by solutions which are too smooth (over regularized). But the vertical part is characterized by solutions dominated by the effects of errors (under regularized). The optimum value of the regularization is at the corner of the curve. At the corner, solutions with smallest residual and limited size will be found. The average calculated L-curve corner was implemented into the Tikhonov formulation to find the corresponding regularized solution.

As a preliminary validation of results, the regularized solutions together with transfer matrices imported into a forward formulation (Eq. (1)) to estimate the responses as presented in Fig. 8. It shows that estimated and measured responses are in agreement however, they are different at some frequencies. They are not expected to be wholly the same because the Tikhonov regularization is a compromise between the solution norm and residue norm. Differences are due to residue norm which was adjusted in order to find the regularized solution. Difference between two plots may be calculated by:

$\left[(A X)_{\text {Experimental }}-(A X)_{\text {Inverse Formulae }}\right]_{f}=\left[(\text { Measured Responses })-(A X)_{\text {Inverse Formulae }}\right]_{f}$

Pressure sensors implemented to measure pressure inside the cylinders during the engine operation. Fig. 9 shows the corresponding time domain excitation on a crankpin. These values converted to frequency domain and together with regularized solutions are shown in Fig. 10. It presents the regularized solutions which L-curve criterion implemented to find the regularization parameter. It shows good agreement between the solutions and the measured values.

\section{CONCLUSIONS AND RECOMMENDATIONS}

Engine internal vibration sources were estimated very well using an inverse formulation. Tikhonov technique conducted to find the regularized solutions in such an illconditioned system. L-curve criterion utilised to find the optimum regularization parameter. This inverse formulation can be considered as the basis of a whole approach to find the internal forces of machinery. The entire mathematical model of the system can be achieved by modeling all the engine parts through their transfer functions. An accessible point should be used for response measurement. Then these responses may be related to any internal excitation (near or far) by a series of transfer functions. Non-linearities corresponding to non-coherent output power of systems may be cited as the shortcoming of this method. 


\section{REFERENCES}

[1] Woodbury, K.A, INVERSE ENGINEERING HANDBOOK, Chapter 2: Sequential function specification method using future times for function estimation, CRC Press, (2003).

[2] Press, W.H., Flannery, B.P., Teukolsky, S.A., and Vetterling, W.T., Numerical recipes--The art of scientific computing (FORTRAN version), Cambridge University Press, 702 p, (1989).

[3] Andersen, M.W., Pedersen J., Localized Solutions in Inverse Sound Source Reconstructions, Polytechnic midway project at IMM, Technical University of Denmark, (2003).

[4] Hansen, P.C., "Regularization Tools: A Matlab Package for Analysis and Solution of Discrete III-Posed Problems", Numerical Algorithms 6: 1-35, (1994).

[5] Hansen, P.C., Rank Deficient and Discrete III-Posed Problems: Numerical Aspects of Linear Inversion, SIAM, Philadelphia, (1998). 


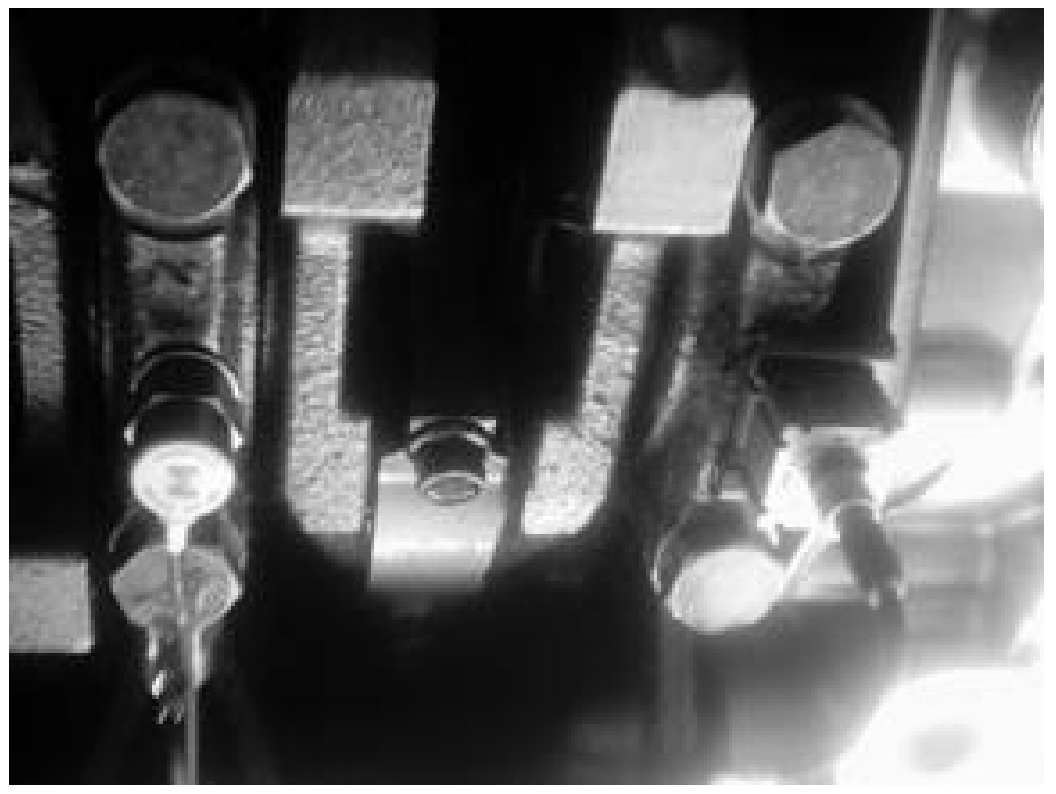

Fig. 1. Photo showing two of the accelerometers mounting on the crankshaft bearings.

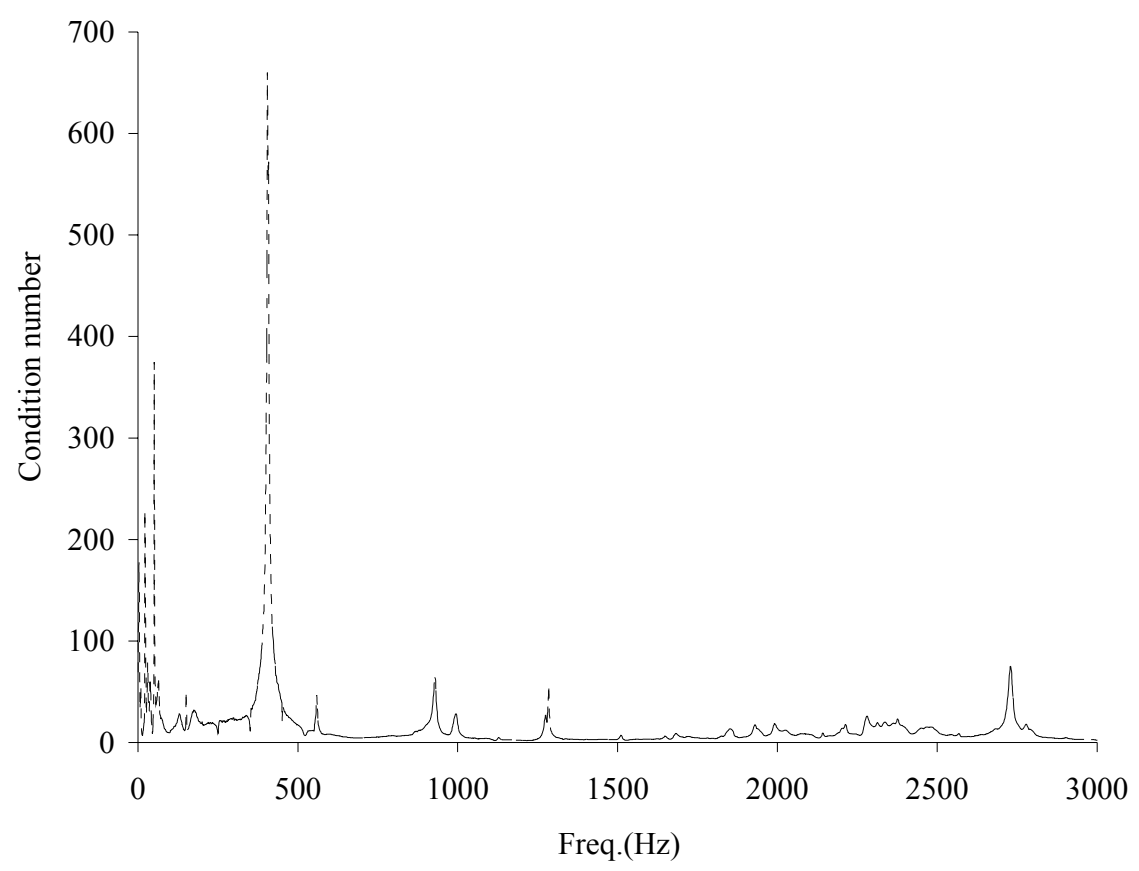

Fig. 2. Condition numbers of transfer functions. 

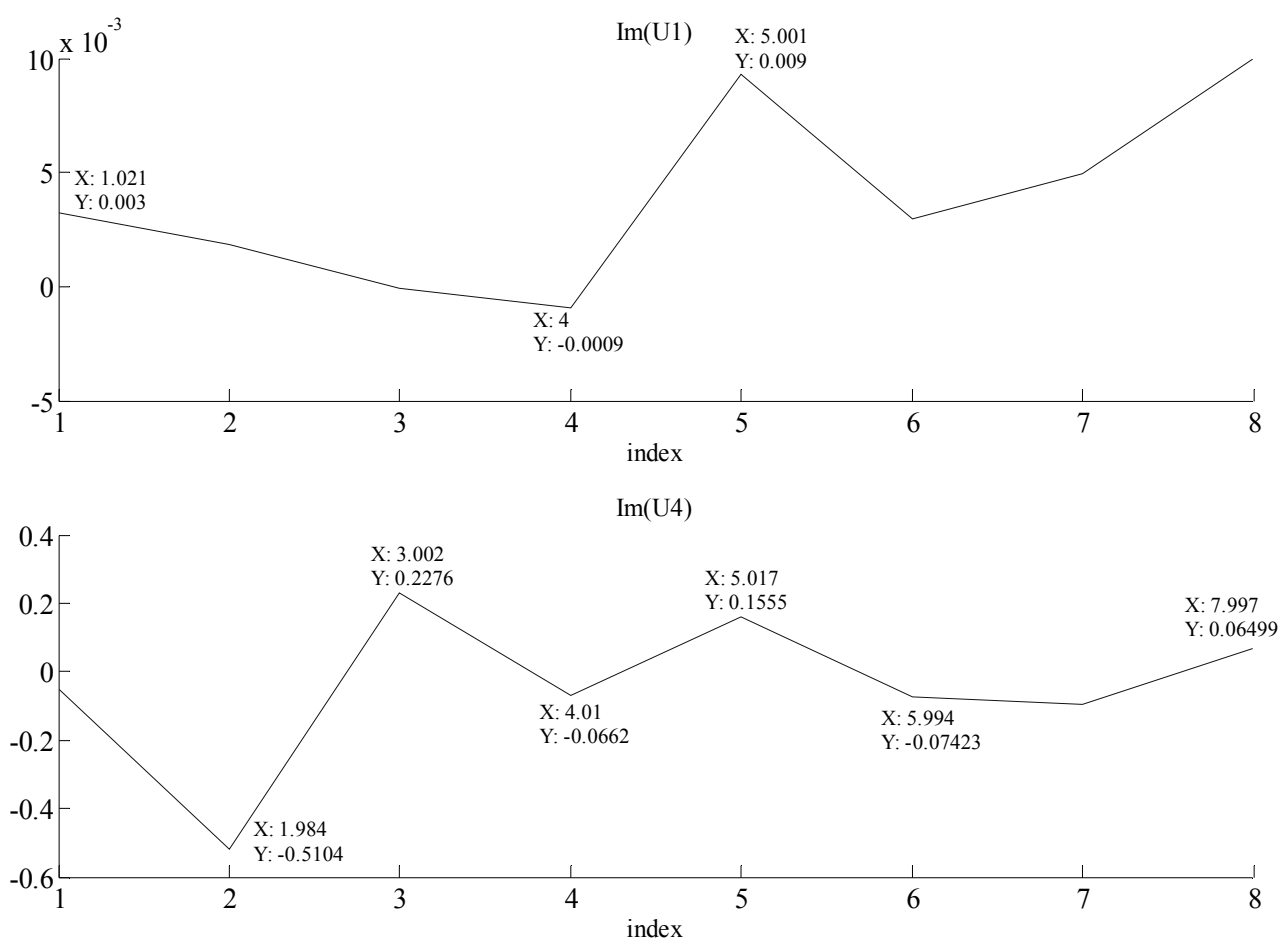

Fig. 3. Sign changes of imaginary parts of the first $\left(U_{1}\right)$ and last $\left(U_{4}\right)$ index of left singular vectors, based on the $8 \times 4$ transfer matrices, $f=100 \mathrm{~Hz}$.

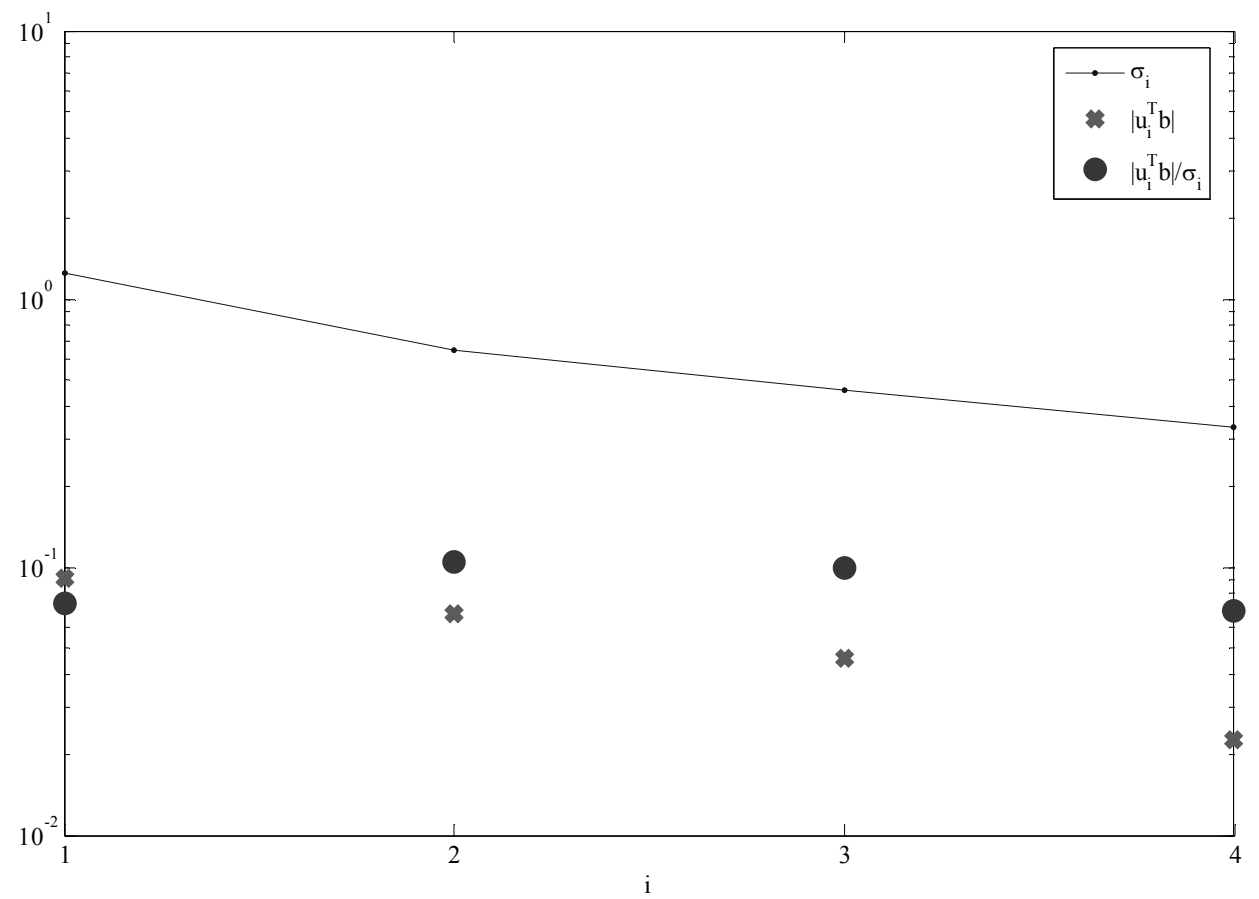

Fig. 4. Picard plot for cylinder\#4, 930(Hz). 


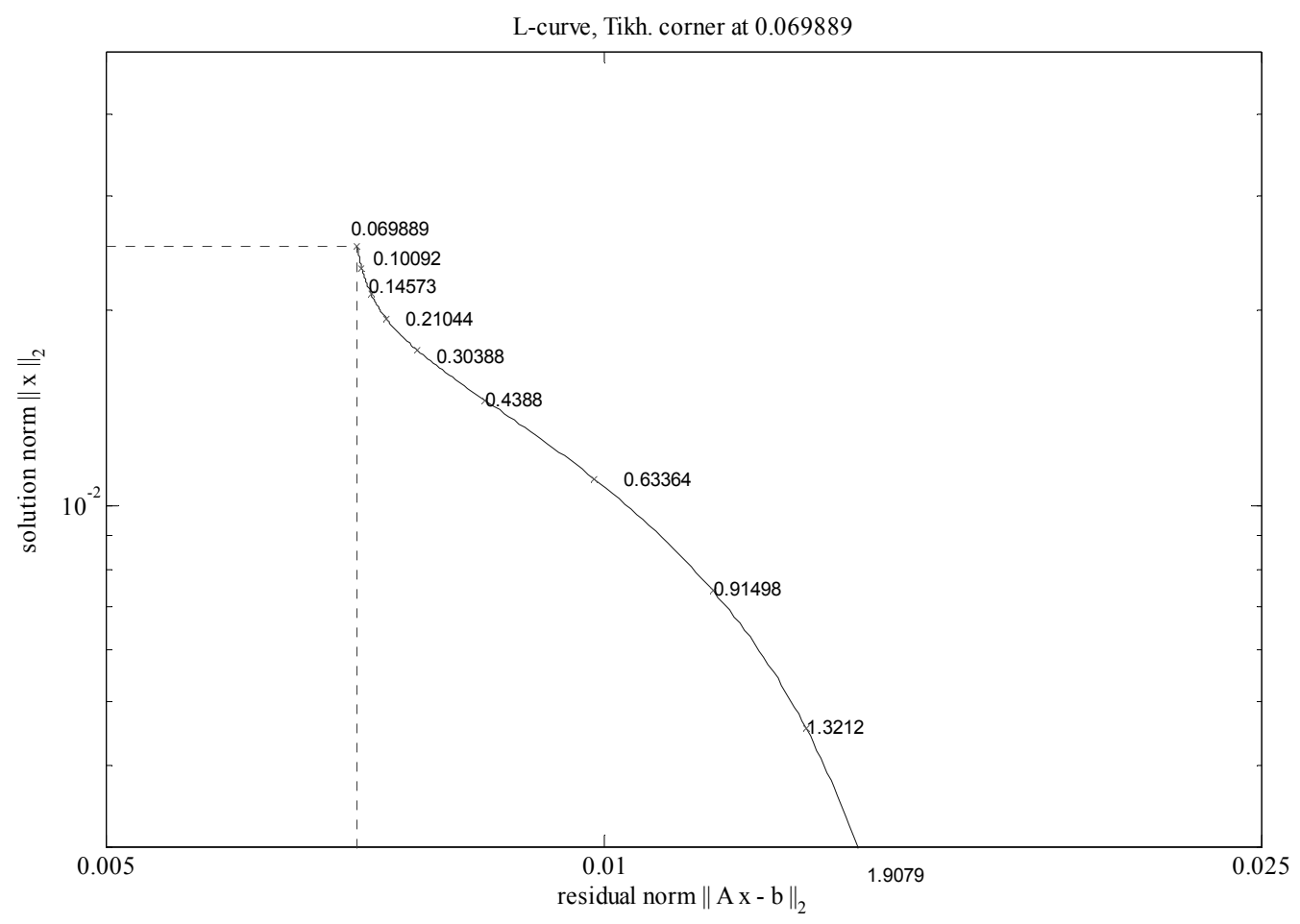

Fig. 5. L-curve plot for cylinder\#4, $930 \mathrm{~Hz}$.

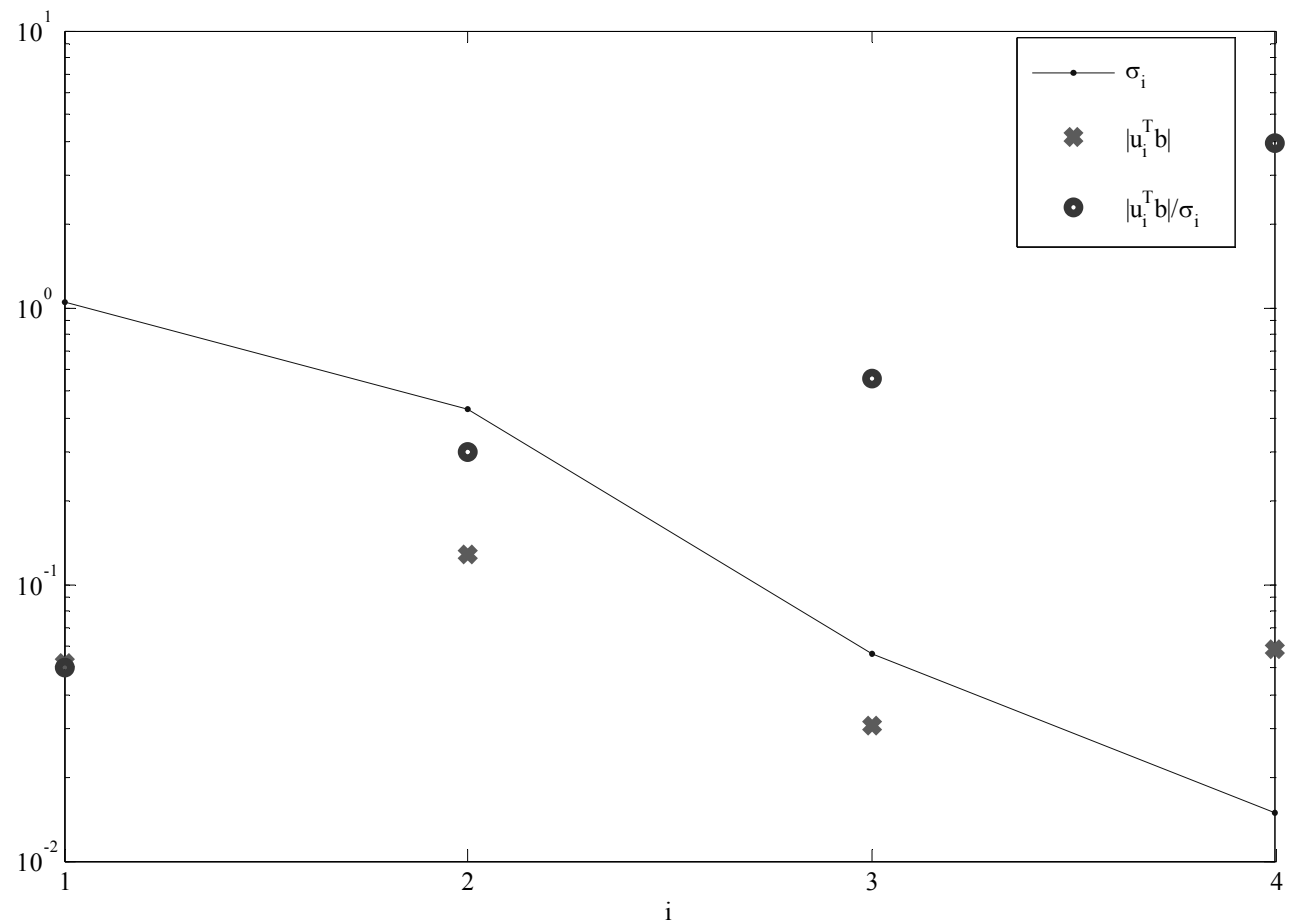

Fig. 6. Picard plot for cylinder\#2, 130(Hz). 


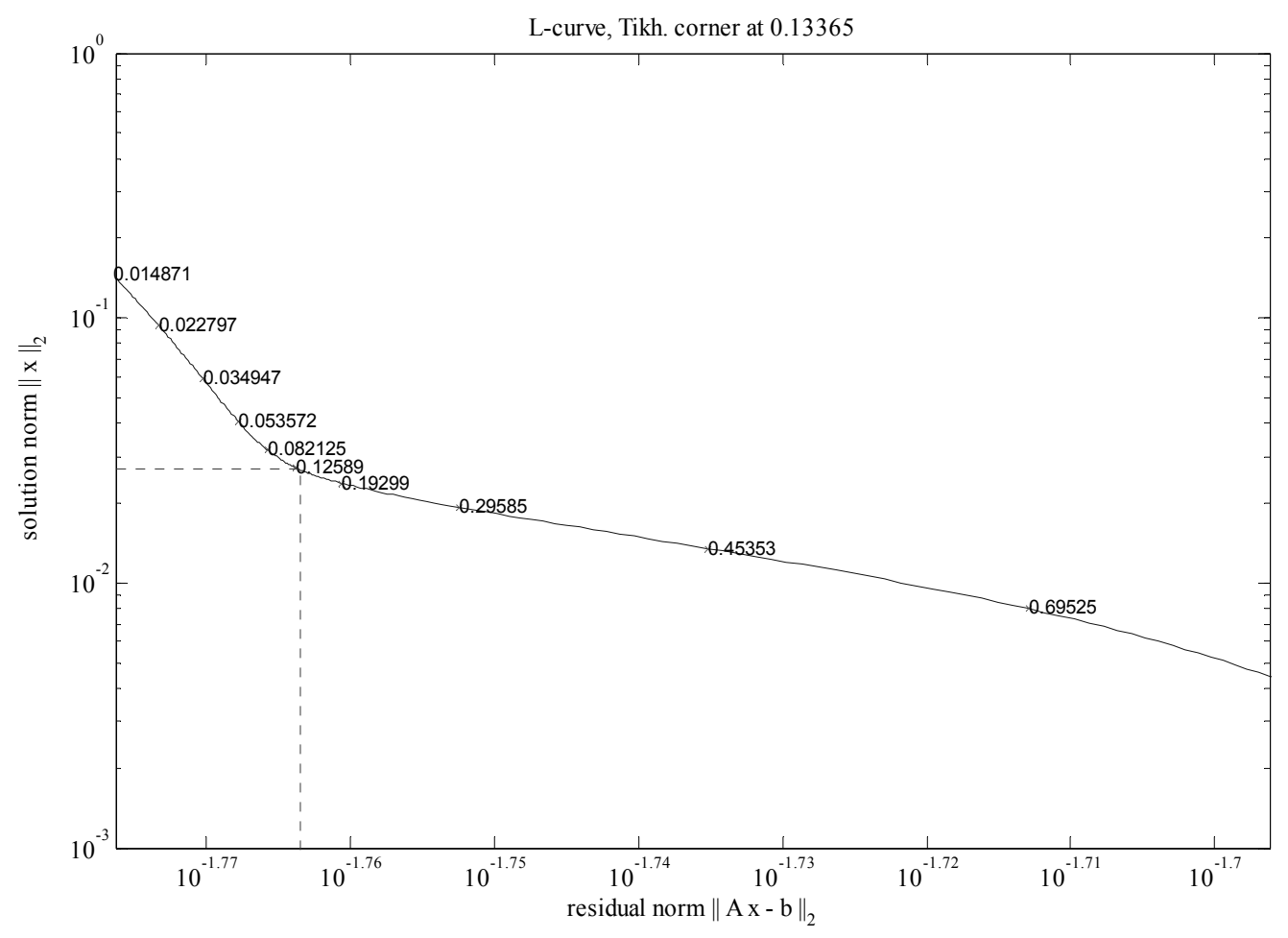

Fig. 7. L-curve plot for cylinder\#2, 130(Hz).

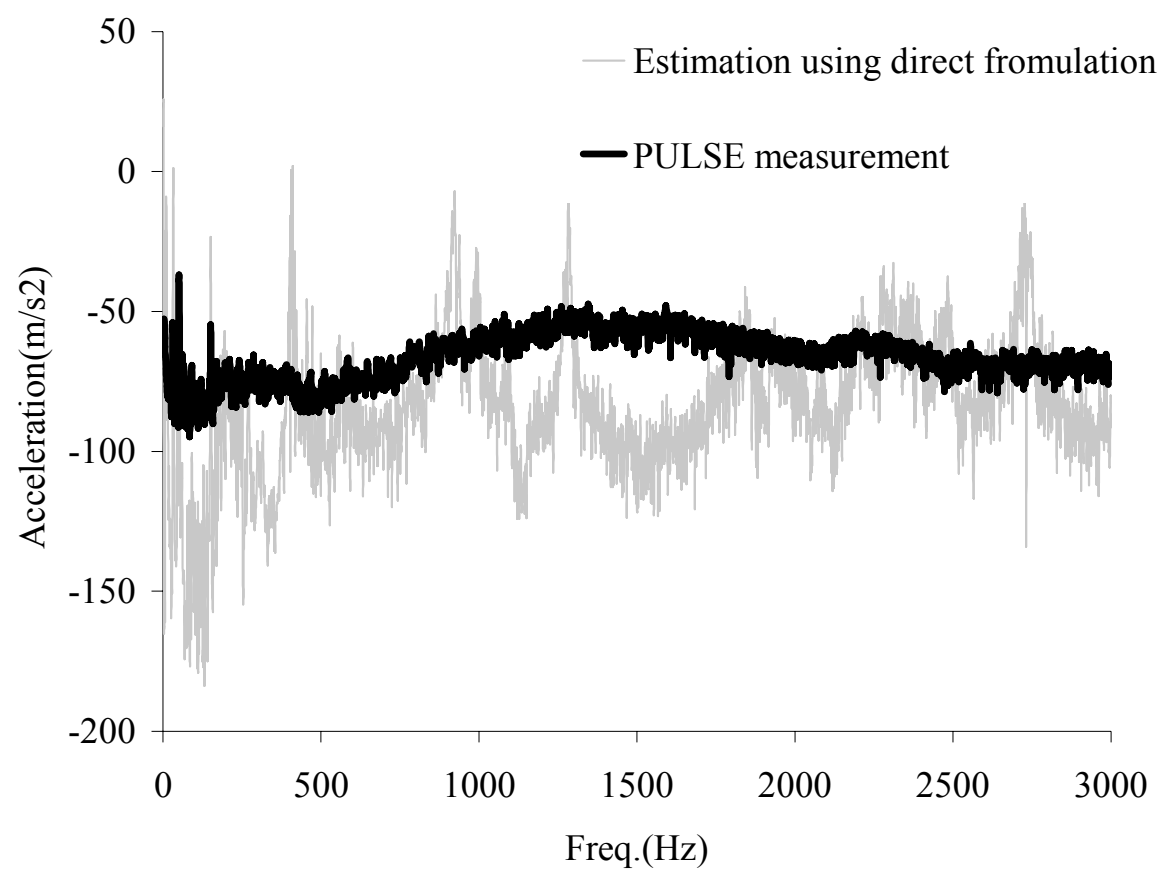

Fig. 8. Comparison between the measured responses and responses estimated by direct formulation. 


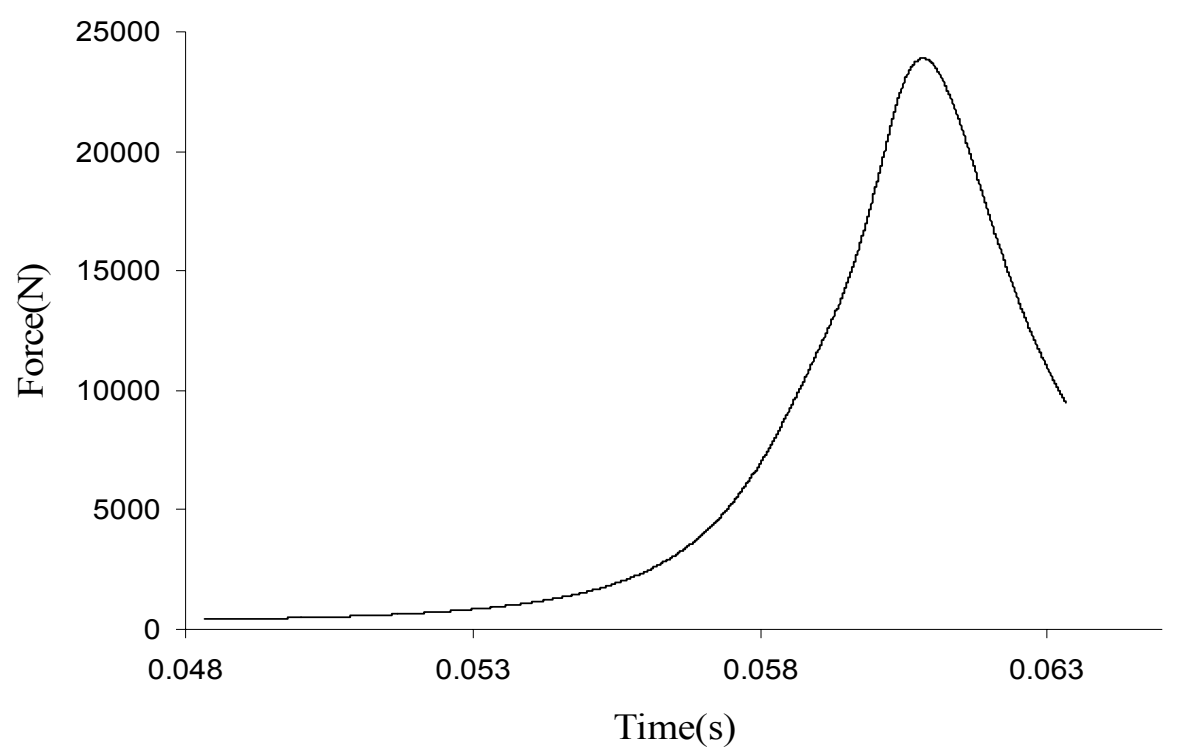

Fig. 9. Piston force applied to a crankpin through the connecting rod.

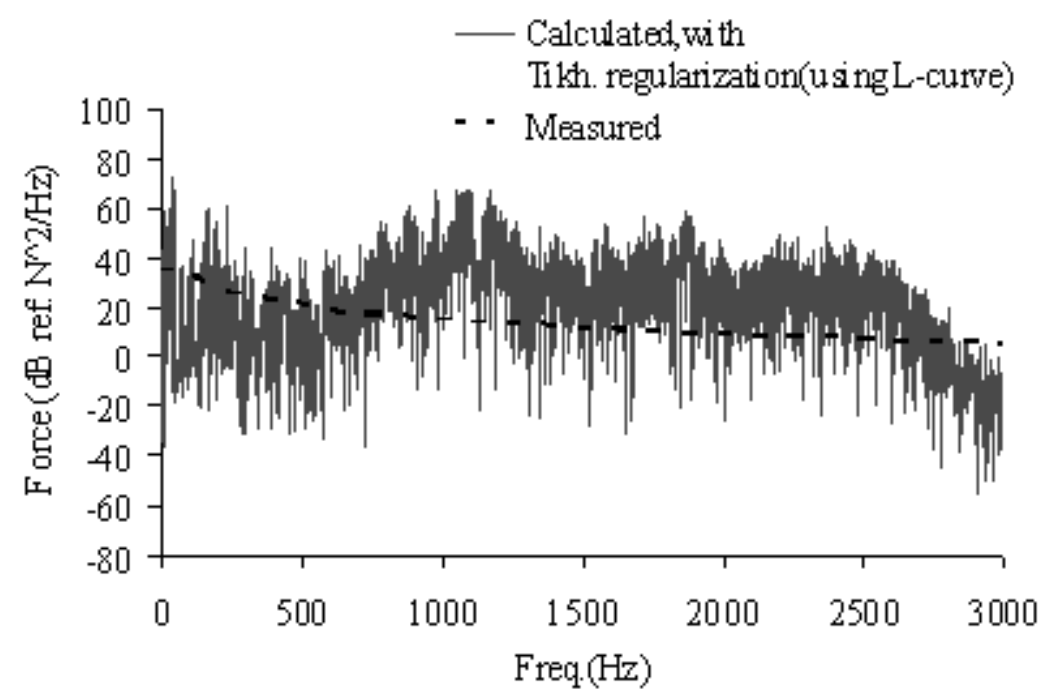

Fig. 10. Inverse estimation of an excitation. 